\title{
Neuroendokrine Tumoren des Gastrointestinaltrakts
}

\author{
Bertram Wiedenmann ${ }^{a} \quad$ Andrea Frilling $^{b}$ \\ ${ }^{a}$ Medizinische Klinik mit Schwerpunkt Hepatologie, Gastroenterologie, Endokrinologie und Diabetes, Charité Universitätsmedizin Berlin - \\ Campus Virchow Klinikum, Berlin, Deutschland \\ ${ }^{\mathrm{b}}$ Department of Surgery and Cancer, Imperial College London, Hammersmith Hospital, UK
}

Die diagnostischen und therapeutischen Angebote für Patienten mit neuroendokrinen Tumoren des gastroenteropankreatischen Systems haben sich in den letzten 10 Jahren wesentlich verbessert. Ziel dieses Schwerpunkthefts ist es, den aktuellen Stand zu den verfügbaren diagnostischen Möglichkeiten einschließlich der hierzu vorliegenden Standards vorzustellen. Das Gleiche gilt auch für neue Therapieoptionen, die das Potential haben, die bisher ungünstige Prognose der Patienten in fortgeschrittenen Tumorstadien positiv zu beeinflussen. Ein besonderen Augenmerk galt dabei der Behandlung von Lebermetastasen, die bei neuroendokrinen Tumoren eine entscheidende prognostische Bedeutung haben.

Die biochemische Diagnose von neuroendokrinen Tumoren basiert auf den Nachweis spezifischer Hormone und der Tumormarker Chromogranin A und 5-Hydroxyindolessigsäure (5-HIES). Der immunhistochemische Tumormarker Ki-67 kommt sowohl im Rahmen der Tumorklassifikation als auch als Prognosefaktor zum Einsatz. Als Goldstandard des morphologischen Tumornachweises hat sich die Somatostatinrezeptor-Szintigraphie (Octreoscan) etabliert. Neue kombinierte PET/CT-Verfahren wie das 68-Ga-DOTATOC-PET/ CT zeichnen sich durch eine sehr hohe Sensitivität aus und sind wegbereitend für eine gegen Somatostatinrezeptoren gerichtete systemische Therapie.

Wegen der häufig bilobären und multifokalen Manifestation von neuroendokrinen Lebermetastasen ist eine Leberresektion mit kurativer Intention nur bei wenigen Patienten möglich. Eine palliative zytoreduktive Resektion ist im Ein- zelfall bei funktionierenden Metastasen und Ineffektivität der medikamentösen Behandlung indiziert. Unter sorgfältiger Beachtung der Selektionskriterien kann auch eine Lebertransplantation in Erwägung gezogen werden.

Angefangen bei dem endlich vorliegenden Nachweis, dass alt bewährte Substanzen auch in unserer Ära der Evidenzbasierten Medizin ihre Wirkung haben, können wir heute mit gutem Gewissen Somatostatinanaloga wie Octreotid auch mit antiproliferativem Behandlungsziel einsetzen. Ergebnisse aktueller Studien belegen die Wertigkeit und Wirksamkeit medikamentöser Wirkstoffe wie Everolimus, Sunitinib, Temozolomid und Streptozotozin.

Lokal ablative Maßnahmen wie die Radiofrequenzablation können sowohl die klinische Symptomatik als auch den Tumorprogress positiv beeinflussen. Unter den perkutanen lebergerichteten Techniken liefert neben der bereits etablierten transarteriellen Chemoembolisation die selektive interne Radiotherapie mit Lutetium-markierten Mikrosphären gute Ergebnisse. Bei Somatostatinrezeptor-positiven Tumoren ist auch die Peptidrezeptor-Radionuklid-Therapie erfolgversprechend, wobei der Stellenwert dieser neuer Therapieoption noch bestimmt werden muss.

Das von den Herausgebern in Zusammenarbeit mit Autoren erarbeitete hier vorliegende Heft gibt eine aktuelle Bestandsaufnahme des State of the Art in der Diagnostik und Therapie neuroendokriner Tumoren des gastroenteropankreatischen Systems.

\begin{tabular}{ll}
\hline KARGER & $\oplus$ o 2010 S. Karger GmbH, Freiburg \\
Fax +497614520714 & Accessible online at: \\
$\begin{array}{l}\text { Information@Karger.de } \\
\text { www.karger.com }\end{array}$ & www.karger.com/vim \\
&
\end{tabular}

Prof. Dr. Andrea Frilling

Department of Surgery and Cancer

Imperial College Healthcare NHS Trust, Hammersmith Hospital

Du Cane Road, London W12 0HS, UK

Tel. +44 203313 32-10, Fax -12

a.frilling@imperial.ac.uk 